\title{
Magnetic anisotropy in Fe phthalocyanine film deposited on Si(110) substrate: Standing configuration
}

\author{
Juan Bartolomé, ${ }^{\text {a) }}$ Fernando Bartolomé, and Luis Miguel García \\ Instituto de Ciencia de Materiales de Aragón and Departamento de Física de la Materia Condensada, \\ CSIC-Universidad de Zaragoza, Zaragoza 50009, Spain
}

Thomas Gredig

Department of Physics, California State University, Long Beach, California 90840-9505, USA

Ivan K. Schuller

Center for Advanced Nanoscience, Department of Physics, University of California San Diego, La Jolla, California 92093-0319, USA

\author{
Julio C. Cezar
}

Laboratório Nacional de Luz Síncrotron (LNLS), Campinas, SP CEP 13083-100, Brazil and European Synchrotron Radiation Facility (ESRF), Grenoble F380543, France

(Submitted February 24, 2017)

Fiz. Nizk. Temp. 43, 1189-1193 (August 2017)

\begin{abstract}
In this contribution we report on the structural and magnetic properties of an Fe phthalocyanine (FePc) thin film deposited on a silicon substrate. The planar FePc molecules order spontaneously in a standing configuration, i.e., with the molecular plane perpendicular to the substrate. The X-ray linear polarized absorption and $\mathrm{x}$-ray magnetic circular dichroism experiments at the $\mathrm{Fe}-L_{2,3}$ edges at $T=6 \mathrm{~K}$ were performed, concluding that at this temperature the film displays magnetic anisotropy with the main easy axis perpendicular to the substrate. This result is explained in terms of the FePc single molecule anisotropy which has its larger moment in the molecule plane. Thus, the standing configuration in polycrystalline thin films favors statistically that, at the macroscopic array level, the magnetic easy anisotropy axis is normal to the substrate. Published by AIP Publishing. [http://dx.doi.org/10.1063/1.5001295]
\end{abstract}

\section{Introduction}

Molecular thin films have a broad field of applications as coatings in optical devices and sensors. In particular, bioinspired oxygen-binding metalated macrocycles, such as iron-phthalocyanines (FePc), are being investigated due to their unique magnetic properties as a molecular magnet. ${ }^{1-3}$ The FePc is a planar molecule where the $\mathrm{Fe}$ atom has a square planar coordination with four pyrrolitic $\mathrm{N}$ atoms (see Fig. 1). When deposited on a planar substrate the resulting configuration depends on the type of substrate and deposition method. Organic molecular beam epitaxy (OMBE) allows producing metallo-phthalocyanine (MPc) $(\mathrm{M}=$ transition metal) films with different crystalline orientations and order. Frequently, the planar MPc molecules stack parallel to the substrate surface (laying configuration), when deposited on $\mathrm{Au},{ }^{4} \mathrm{Ag}^{5-7}$ or $\mathrm{Cu},{ }^{8}$ or tilted with the MPc plane nearly perpendicular to the substrate plane (standing configuration), as for FePc deposited on sapphire ${ }^{4,9-11}$ or silicon, ${ }^{11,12}$ on polycrystalline substrates ${ }^{13}$ or low work function metals as $\mathrm{Al}$ and $\mathrm{V}^{14}$

In previous work ${ }^{2}$ we found that FePc films deposited on $\mathrm{Au}$, ordered in the laying configuration and had magnetic planar anisotropy, i.e., with the magnetic easy axis parallel to the molecule plane, thus parallel to the substrate. This was quite surprising in view of the magnetic behavior in the bulk phase. ${ }^{15}$ The most exiciting result was the observation by $\mathrm{x}$-ray magnetic circular dichroism (XMCD) of a large unquenched orbital moment caused by the orbital degeneracy of the $e_{g}$ doublet level near the Fermi energy. ${ }^{2}$

The objective of this paper is to describe the magnetic anisotropy of a FePc thin film deposited by OBME on a Si(110) substrate, thus in the standing configuration, and compare results with the $\mathrm{FePc}$ film deposited on $\mathrm{Au}$ in the laying configuration.

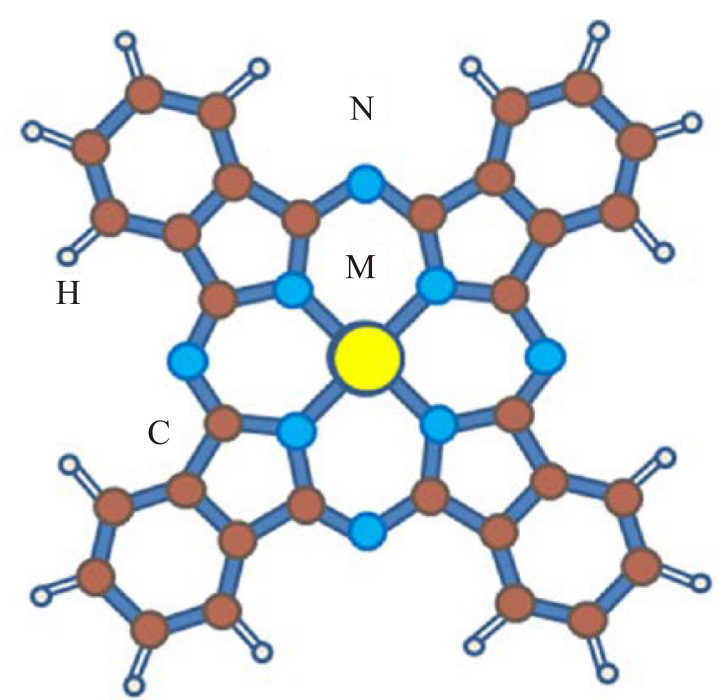

Fig. 1. Metallo-phthalocyanine molecule. 

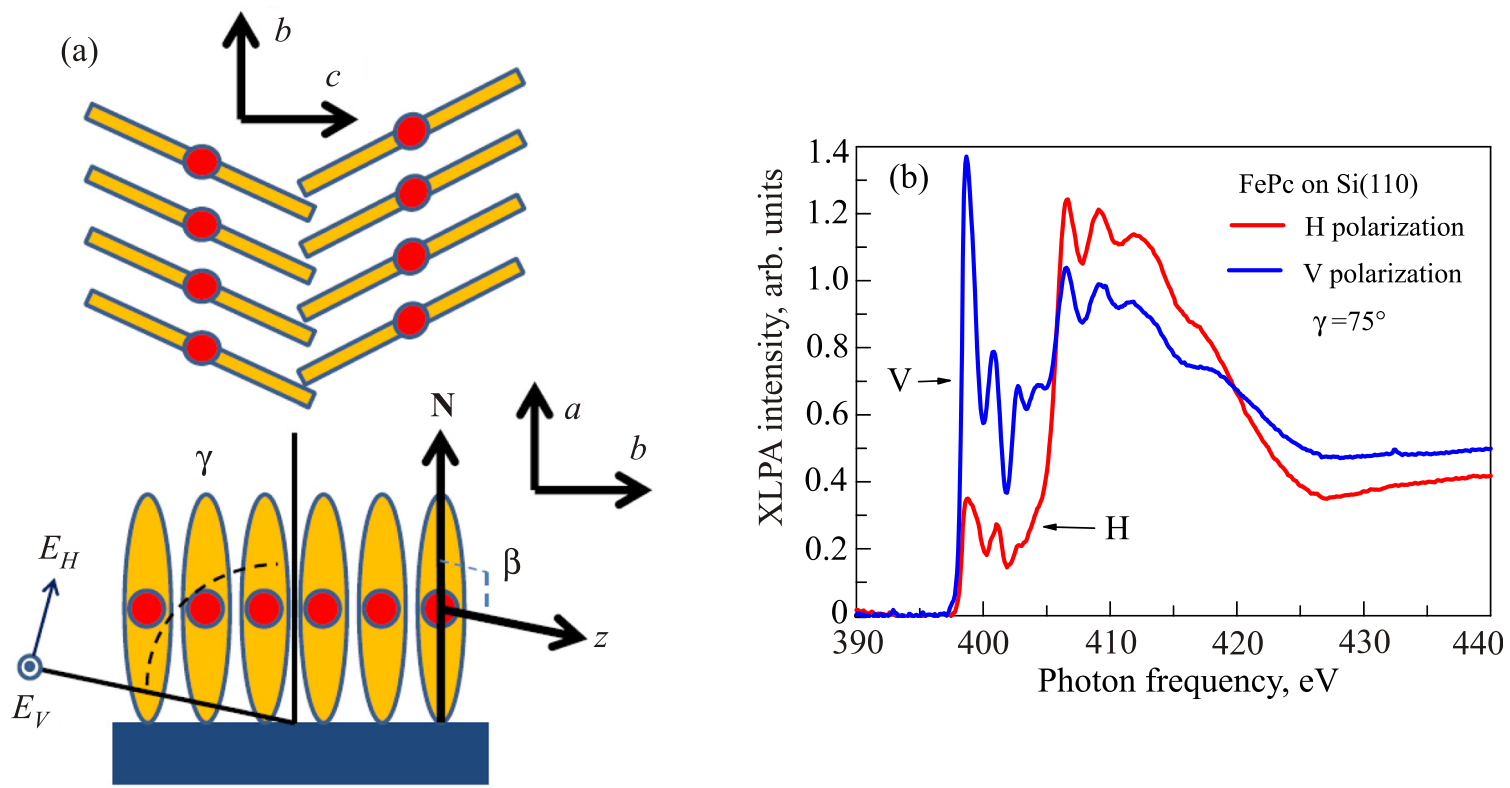

Fig. 2. (a) Side view of the FePc stacking on a $\mathrm{Si}(110)$ substrate in the standing configuration. The incoming beam at grazing incidence $\gamma$ is shown with the $\mathbf{E}$ in the $\mathrm{H}$ and $\mathrm{V}$ polarization modes, at $T=6 \mathrm{~K}$. The angle $\beta$ between the molecule $z$ axis and the normal to the substrate $\mathbf{N}$. Upper panel: top projection of the chains on the substrate. (b) XLPA spectra recorded at the $\mathrm{N} K$-edge in grazing angle incidence $\gamma=75^{\circ}$. The two spectra correspond to the $\mathrm{H}$ and $\mathrm{V}$ polarization modes: $\mathbf{E}$ is either (blue, V) close to parallel to the silicon substrate, or (red, $\mathrm{H}$ ) forming an angle $15^{\circ}$ with $\mathbf{N}$.

\section{Experimental details}

The FePc thin film was deposited on a $\mathrm{Si}(110)$ substrate by OMBE. Sigma-Aldrich FePc was purified in three cycles using the temperature gradient method. Outgassing the purified material was found essential to reduce the number of impurities and increase the crystallinity. The chamber pressure during deposition was $1.2 \times 10^{-8}$ Torr with the base pressure about $5 \times 10^{-9}$ Torr (more details in Ref. 12). Depending on the substrate temperature during deposition, asymmetrical, elongated grains are formed. Between room temperature and $200^{\circ} \mathrm{C}$ the grains consist of FePc molecules stacked in the form of chains, with an approximately $26.5^{\circ}$ tilting angle with respect to the chain axis $b$ (the $\alpha$ phase). The chain direction $b$ is parallel to the substrate plane $e^{9,16}$ [see Fig. 2(a)]. The measured sample had a FePc thickness of about $80 \mathrm{~nm}$.

The standing configuration of the FePc molecules was checked by $\mathrm{x}$-ray diffraction $(\theta-2 \theta$ diffraction patterns) where the (200), (400) and (800) peaks of the FePc textured sample have highest intensity (see Fig. 1 in Ref. 12). In addition, we have performed an X-ray linear polarized absorption (XLPA) experiment at the $\mathrm{N} K$-edge at normal and grazing incidence $\left(75^{\circ}\right.$ from normal) [see Fig. 2(a)]. As shown in this figure, the polarization of the electric field of the incoming photons $\mathbf{E}$ is denoted as horizontal $(\mathrm{H})$ and vertical (V) for parallel and perpendicular to the synchrotron storage ring electron orbit plane, respectively. In Fig. 2(b), the two spectra are shown: it is evident that both are very different, with the most intense signal corresponding to the $\mathrm{V}$ configuration. This is in quantitative agreement with earlier measurements on the laying configuration, as shown in Fig. 2, Ref. 2. These measurements therefore confirm that in the sample discussed here, the FePc molecules are oriented in the standing configuration.

The XLPA and XMCD experiments were performed at the ID08 beamline of the European Synchrotron Radiation Facility (ESRF) in Grenoble. The detection mode was total electron yield (TEY). The resolution at the Fe- $L_{2,3}$ energy region is about $\Delta E / E=5 \times 10^{-4}$. The monochromatic $\mathrm{x}$-ray beam incidence angle on the sample may be varied in the range $0<\gamma<75^{\circ}$. The XMCD experiment was performed in a $\mu_{0} H=5 \mathrm{~T}$ applied magnetic field, parallel to the beam direction. The polarization rate was considerably above 99\%. All experiments were performed at $T=6 \mathrm{~K}$.

\section{XLPA and XMCD results}

The normalized XLPA spectra in the $\mathrm{V}$ polarization mode, measured at the $\mathrm{Fe}-L_{2,3}$ edges are shown in Fig. 3. The absorption intensity is proportional to the number of empty valence states in the direction of the electric field $\mathbf{E}_{H}$, which is parallel to the substrate plane for $\gamma=0$, and almost perpendicular to it for grazing incidence $\gamma=75^{\circ}$ : The molecules are in the standing configuration, with the $z$ axis of the molecules randomly oriented around the normal to the substrate $\mathbf{N}$ [see Fig. 2(a)]. Therefore, the vector $\mathbf{E}_{H}$, acting as

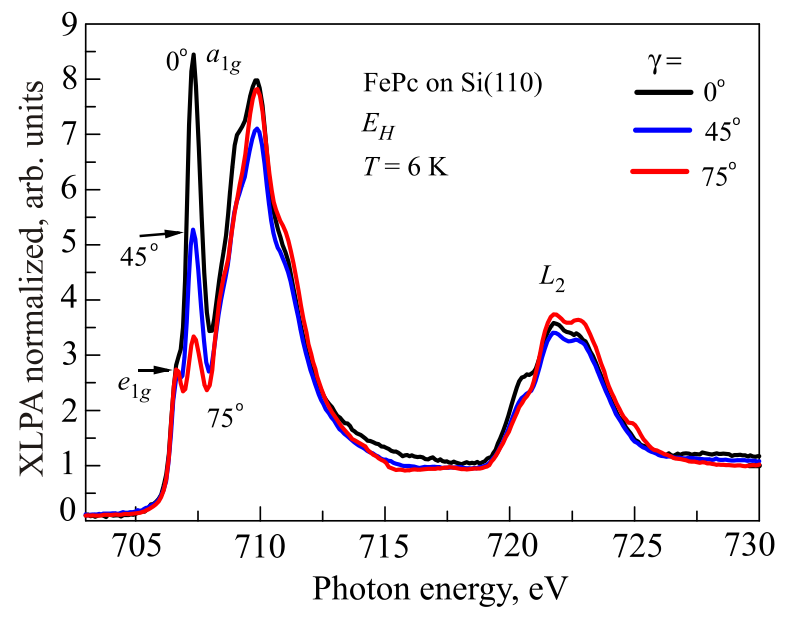

Fig. 3. Normalized XLPA of FePc deposited on $\mathrm{Si}(110)$ at the $\mathrm{Fe}-L_{2,3}$ edges as a function of incident angle $0<\gamma<75^{\circ}$ measured at $T=6 \mathrm{~K}$. 
"search light," scans a random distribution of the molecule orientations between the $z$ and $x$ (or $y$ ) axes for $\gamma=0$, and mostly $y$ (or $x$ ) axis for $\gamma=75^{\circ}$, the largest grazing angle possible in the experiment.

By direct comparison to the XLPA spectra measured in the laying configuration we can establish a direct correspondence between the energy of the peaks observed in both spectra, and as a consequence, the identification of some of them to $2 p \rightarrow 3 d$ excitations. $^{2}$ The first small peak at 706.4 $\mathrm{eV}$ observed at the L3 edge in the $\gamma=75^{\circ}$ spectrum can be assigned to the $e_{g}$ empty level with mixing of the a1g level; this is a very interesting feature, specific to the FePc, which is not present in other MPc's $(\mathrm{M}=\mathrm{Cu}$, Ni or Co). This reveals the existence of a split doublet near the Fermi energy which causes the highly un-quenched orbital moment. ${ }^{2}$ The next intense peak at $707.3 \mathrm{eV}$ corresponds to the excitations to the empty $a_{1 g}$ state (or to the $d_{3 z^{2}-r^{2}}$ state), which is most intense for $\gamma=0$, since in this incident angle the molecular $z$ axis has the maximal projection on the $\mathbf{E}_{H}$ vector. The photon energy of the peak corresponds exactly to the values observed for the FePc film deposited on $\mathrm{Au}$ in the laying configuration. $^{2}$ However, the dependence of the intensity with respect to the incident angle is just reversed, i.e., maximal peak for standing configuration corresponds to minimal intensity for laying configuration, and vice versa.

The x-ray absorption spectra (XAS) (not shown) and XMCD spectra measured at the $\mathrm{Fe}-L_{2,3}$ edges in a field of $\pm 6 \mathrm{~T}$ as a function of incident angle are depicted in Fig. 4. The sum rules analysis ${ }^{17,18}$ was applied to the XMCD to obtain the effective spin $m_{S}^{\text {eff }}$ and orbital $m_{L}$ moments as a function of $\gamma$. The results are shown Fig. 5.

\section{Discussion}

The XMCD experiment, performed in a magnetic field parallel to the incoming $\mathrm{X}$-ray beam at an incident angle $\gamma$, provides the projection of the Fe magnetic moment along the direction of the magnetic field $m(\gamma)=\mathbf{H} \cdot \mathbf{m} / H$ The FePc molecule symmetry may be approximated to $D_{4 h}$, thus the molecular $z$ axis corresponds to the fourfold axis, while the two perpendicular axes $x$ and $y$, are assumed to be identical.

In what follows we assume the intermolecular magnetic interactions to be negligible. Our sample in the present work can be modeled as consisting of chains of parallel stacked

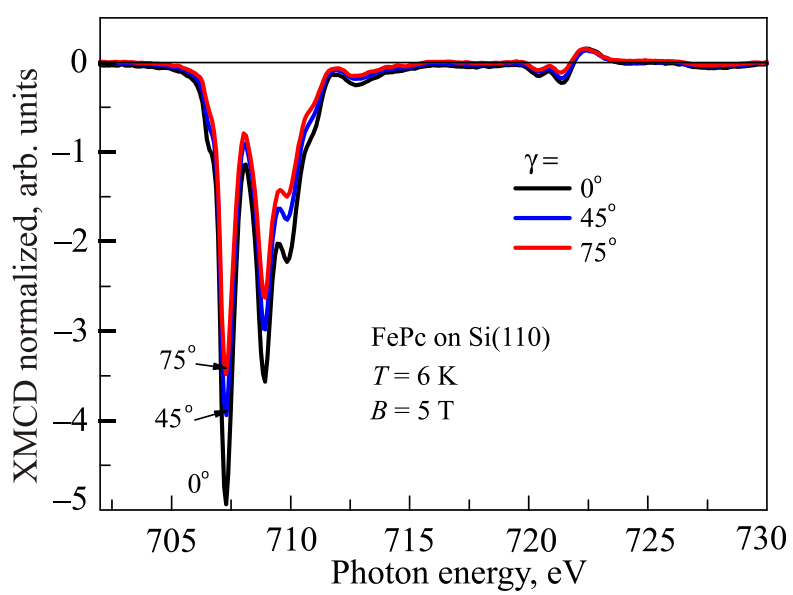

Fig. 4. Normalized XMCD of FePc deposited on $\mathrm{Si}(110)$ at the $\mathrm{Fe}-L_{2,3}$ edges as a function of incident angle $0<\gamma<75^{\circ}$ measured at $T=6 \mathrm{~K}$.

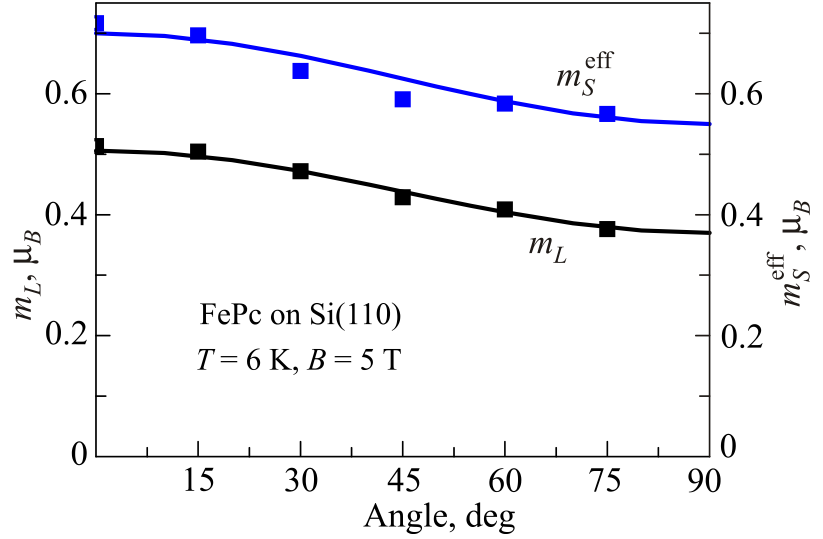

Fig. 5. $m_{L}$ and $m_{S}^{\text {eff }}$ as determined from the XMCD spectra at $T=6 \mathrm{~K}$ and $B=5 \mathrm{~T}$. The parameters used to obtain the fits to Eq. (2) are collected in Table 1.

molecules along the $b$ axis, with their $z$ axis tilted by $26.5^{\circ}$ with respect to the $b$ axis. The chains are parallel to the substrate [see Fig. 2(a)] and form structural domains, which give rise to a well defined x-ray diffraction pattern. ${ }^{4}$ The direction of the chain $b$ axes are distributed according to the mosaicity of the substrate. Thus we assume a statistically random distribution of $b$ axes in angle around the normal to the substrate $\mathbf{N}$, but always parallel to the substrate [see Fig. 2(a)]. In addition, each molecular plane forms an angle within the range $63.5^{\circ}<\beta<90^{\circ}$ with respect to $\mathbf{N}$, depending on the orientation of the chain $a$ axis. For completeness sake, in Eq. (1) we show the statistical average of the magnetization as a function of incident angle for a fixed $\beta$ angle.

Using $m_{L}=-\left\langle L_{z}\right\rangle \mu_{B} / \hbar$ the orbital moment and $m_{S}^{\text {eff }}$ $=m_{S}-7 m_{T}$, where $m_{S}=-2\left\langle S_{z}\right\rangle \mu_{B} / \hbar$ is the spin moment and $m_{T}=-2\langle T\rangle \mu_{B} / \hbar$ is the intra-atomic dipolar moment, one obtains

$$
\begin{aligned}
m(\gamma)= & \left(m^{y} \sin ^{2} \beta+m^{z} \cos ^{2} \beta\right) \cos ^{2} \gamma \\
& +\frac{1}{2}\left(m^{x}+m^{y} \cos ^{2} \beta+m^{z} \sin ^{2} \beta\right) \sin ^{2} \gamma .
\end{aligned}
$$

The angle between the molecular $z$ axis and $\mathbf{N}$ is $\beta \approx 90^{\circ}$, while maintaining the herringbone structure on the plane parallel to the substrate [see Fig. 2(a)]. ${ }^{4}$ With the approximation $m^{x}=m^{y}$, Eq. (1) becomes

$$
m(\gamma)=m^{x} \cos ^{2} \gamma+\frac{1}{2}\left(m^{x}+m^{z}\right) \sin ^{2} \gamma
$$

The film average orbital magnetic moment per $\mathrm{Fe}$ atom is larger for normal incidence than in the direction of the substrate plane $\left(m_{L}^{\perp}>m_{L}^{\|}\right)$, thus the film with standing configuration has a film anisotropy perpendicular to the substrate, in contrast to the laying configuration which is parallel to the substrate $\left(m_{L}^{\perp}>m_{L}^{\|}\right)$.

TABLE 1 . The four magnetic moment parameters in units of $\mu_{B}$ determined with respect to the directions $\mathbf{N}\left(m^{\perp}\right)$ and parallel to the substrate $\left(m^{\|}\right)$for the FePc films in standing and laying configurations.

\begin{tabular}{lcccc}
\hline \hline Configuration & $m_{L}^{\|}$ & $m_{L}^{\perp}$ & $m_{S}^{\text {eff, } \|}$ & $m_{S}^{\text {eff, } \perp}$ \\
\hline FePc on Si(110) standing conf. & $0.37(5)$ & $0.50(4)$ & $0.55(5)$ & $0.70(5)$ \\
FePc on Au laying conf. & $0.53(5)$ & $0.29(4)$ & $0.69(5)$ & $0.10(5)$ \\
\hline \hline
\end{tabular}


TABLE 2. First entry: the four adjusted magnetic moment parameters in units of $\mathrm{m}_{B}$, obtained from the fit of the XMCD sum rule results to Eq. (2), and calculated orbital to spin moment ratio. Second entry: same parameters for FePc film deposited in the laying configuration. ${ }^{2}$

\begin{tabular}{lllllll}
\hline \hline Configuration & $m_{L}^{z}$ & $m_{L}^{x y}$ & $m_{S}$ & $m_{T}^{z}$ & $m_{L}^{x y} / m_{S}$
\end{tabular}

FePc on Si(110) Standing conf. $0.24(5) \quad 0.50(4) \quad 0.59(5) \quad 0.027(5) \quad 0.86(4)$ $\begin{array}{llllll}\text { FePc on Au Laying conf. } \quad 0.29(5) & 0.53(4) & 0.64(5) & 0.074(5) & 0.83(4)\end{array}$

The data in Fig. 5 have been fitted to Eq. (2) for each component, orbital or effective spin. The resulting moment components are collected in Tables 1 and 2.

The values of the single molecule magnetic moment parameters in the standing configuration are very similar to those found for the laying one. Actually, the orbital to spin ratio $m_{L}^{x y} / m_{S}$ is the same for both configurations, within experimental errors. This is an important result since it indicates that the orbital moment is very large, stemming from unquenched orbital moment due to the orbital degeneracy of the $e_{g}$ doublet at the Fermi surface. ${ }^{2}$ We note here that the maximum orbital magnetic moment $m_{L}^{z} \approx 0.50(4)$ is several times larger than predicted from DFT calculations. ${ }^{19}$

Besides, the intra-atomic magnetic dipolar component differs between the two configurations by a factor close to three. This difference is probably related to the differences in the position of the $\mathrm{Fe}$ atom and orientation of each molecule with respect to their adjacent ones.

In this respect, the chains in the standing configuration order within the chain in the $\alpha$-phase type of herringbone structure, in which the Fe atom in one molecule is near the center of one of the pyrrolic rings of the adjacent molecule at each side. ${ }^{4}$ On the other hand, we proved earlier by scanning tunneling microscopy and $\mathrm{x}$-ray absorption at the $\mathrm{Fe}-\mathrm{K}$ edge that in the laying configuration each $\mathrm{Fe}$ atom has, as nearest neighbor atom in the adjacent molecules, the $\mathrm{N}$ atom of the pyrrolic ring closest to the central $\mathrm{Fe}$ (so called TF phase).$^{20}$ Therefore, the first coordination of $\mathrm{Fe}$ is different in the two configurations, which explains the very different $m_{T}^{z}$ derived from experiment.

It is interesting to note that the single ion planar anisotropy caused by spin-orbit coupling is actually enhanced in the standing configuration. Indeed, from the values for the orbital and dipolar moments, following Refs. 21 and 22, one may calculate the spin-orbit contribution to the anisotropy energy

$$
\Delta E_{S O}=-\frac{G}{H}\left\{\frac{\xi_{3 d}\left(m_{L}^{z}-m_{L}^{x y}\right)}{4 \mu_{B}}+\frac{\xi_{3 d}^{2}\left[\frac{21 \times 3}{2 \times 2} m_{T}^{z}+A\right]}{\Delta E_{\mathrm{ex}} \mu_{B}}\right\}
$$

with $G / H \approx 0.2$ for transition metals, $\xi_{3 d}=0.05 \mathrm{eV}$ is the spin-orbit coupling constant in $\mathrm{Fe}, \Delta_{\mathrm{ex}}=0.05 \mathrm{eV}$ is the energy shift between the majority and minority states, and $A$ negligible, as described in Ref. 2, one obtains $\Delta E_{S Q}=7$ $\times 10^{-5}$ and $4.5 \times 10^{-4} \mathrm{eV} / \mathrm{Fe}$ atom for the laying and standing configurations, respectively. That is, the planar anisotropy (positive $\Delta E_{S Q}$ ) is actually enhanced by spin-orbit coupling in the standing configuration with respect to the laying one.

\section{Conclusions}

The present results for the standing configuration confirm the extraordinarily large unquenched orbital moment of FePc single molecules due to the orbital degeneracy of the $e_{g}$ doublet near the Fermi surface that was found in the laying configuration. The FePc single molecule magnetic anisotropy in the standing configuration is planar and nearly identical to that found for the FePc in the laying configuration. In contrast, the standing configuration differs from the laying one in showing perpendicular anisotropy as a film. This is caused by the single ion FePc magnetic planar anisotropy and the statistical average of the molecule moments when the molecule planes are perpendicular to the substrate in the standing configuration.

The financial support of the Spanish MINECO MAT2014-53921-R and Aragonese DGA-IMANA E34, both cofunded by Fondo Social Europeo and European Union FEDER funds is acknowledged. T.G. acknowledges support from the National Science Foundation (NSF) DMR0847552 and the W.M. Keck Foundation. The XMCD experiments were performed at the ID08 (now ID32) beamline of the ESRF, experiment HE2486. This is a highly collaborative research. The experiments were conceived jointly, the data was extensively debated and the paper was written by multiple iteration between all the coauthors. Samples were fabricated, characterized and prepared at UCSD (T.G. and I.K.S.). The research at UCSD was supported by the Office of Basic Energy Science, U.S. Department of Energy, BES-DMS funded by the Department of Energy's Office of Basic Energy Science, DMR under Grant No. DE FG02 87ER-45332.

a)Email: Barto@unizar.es

\footnotetext{
${ }^{1}$ M. Evangelisti, J. Bartolomé, L. J. de Jongh, and G. Filoti, Phys. Rev. B 66, 144410 (2002).

${ }^{2}$ J. Bartolomé, F. Bartolomé, L. M. García, G. Filoti, T. Gredig, C. N. Colesniuc, I. K. Schuller, and J. C. Cezar, Phys. Rev. B 81, 195405 (2010).

${ }^{3}$ J. Bartolomé, C. Monton, and I. K. Schuller, "Magnetism of metal phthalocyanines," in Molecular Magnets: Physics and Applications, edited by J. Bartolomé, F. Luis, and J. F. Fernández (Springer, Berlin, 2014), p. 221.

${ }^{4}$ T. Gredig, K. P. Gentry, C. N. Colesniuc, and I. K. Schuller, J. Mater. Sci. 45, 5032 (2010).

${ }^{5}$ T. Takami, C. Carrizales, and K. W. Hipps, Surf. Sci. 603, 3201 (2009).

${ }^{6}$ K. Manandhar, K. T. Park, S. Ma, and J. Hrbek, Surf. Sci. 603, 636 (2009).

${ }^{7}$ A. Mugarza, R. Robles, C. Krull, R. Korytár, N. Lorente, and P. Gambardella, Phys. Rev. B 85, 155437 (2012).

${ }^{8}$ E. Annese, J. Fujii, I. Vobornik, and G. Rossi, J. Phys. Chem. C 115, 17409 (2011).

${ }^{9}$ C. Miller, A. Sharoni, G. Liu, C. Colesniuc, B. Fruhberger, and I. Schuller, Phys. Rev. B 72, 104113 (2005).

${ }^{10}$ K. P. Gentry, T. Gredig, and I. K. Schuller, Phys. Rev. B 80, 174118 (2009).

${ }^{11}$ T. Gredig, C. N. Colesniuc, S. A. Crooker, and I. K. Schuller, Phys. Rev. B 86, 14409 (2012).

${ }^{12}$ P. D. Ekstrand, D. J. Javier, and T. Gredig, Phys. Rev. B 95, 14406 (2017).

${ }^{13}$ H. Peisert, T. Schwieger, J. M. Auerhammer, M. Knupfer, M. S. Golden, J. Fink, P. R. Bressler, and M. Mast, J. Appl. Phys. 90, 466 (2001).

${ }^{14}$ C. Monton, I. Valmianski, and I. K. Schuller, Appl. Phys. Lett. 101, 133304 (2012).

${ }^{15}$ G. Filoti, M. D. Kuz'min, and J. Bartolomé, Phys. Rev. B 74, 134420 (2006).

${ }^{16}$ G. Liu, T. Gredig, and I. K. Schuller, Europhys. Lett. 83, 56001 (2008).

${ }^{17}$ B. T. Thole, P. Carra, F. Sette, and G. Van Der Laan, Phys. Rev. Lett. 68, 1943 (1992).
} 
${ }^{18}$ P. Carra, B. T. Thole, M. Altarelli, and X. Wang, Phys. Rev. Lett. 70, 694 (1993).

${ }^{19}$ J. Wang, Y. Shi, J. Cao, and R. Wu, Appl. Phys. Lett. 94, 122502 (2009).

${ }^{20}$ F. Bartolomé, O. Bunau, L. M. García, C. R. Natoli, M. Piantek, J. I. Pascual, I. K. Schuller, T. Gredig, F. Wilhelm, A. Rogalev, and J. Bartolomé, J. Appl. Phys. 117, 17A735 (2015).
${ }^{21}$ G. Van Der Laan, J. Phys.: Condens. Matter 10, 3239 (1999).

${ }^{22}$ K. Mamiya, T. Koide, Y. Ishida, Y. Osafune, A. Fujimori, Y. Suzuki, T. Katayama, and S. Yuasa, Radiat. Phys. Chem. 75, 1872 (2006).

This article was published in English in the original Russian journal. Reproduced here with stylistic changes by AIP Publishing. 